\title{
hERGI positivity and Glut-I negativity identifies high-risk TNM stage I and II colorectal cancer patients, regardless of adjuvant chemotherapy
}

This article was published in the following Dove Press journal:

OncoTargets and Therapy

14 October 2016

Number of times this article has been viewed

Leonardo Muratori, ',*

Giulia Petroni, 1,* Lorenzo

Antonuzzo, ${ }^{2,3}$ Luca Boni, ${ }^{4}$

Jessica lorio,, ${ }^{1,3}$ Elena Lastraioli,'

Gianluca Bartoli,' Luca

Messerini,' Francesco Di

Costanzo, ${ }^{2}$ Annarosa Arcangeli

'Department of Experimental and Clinical Medicine, University of Florence, ${ }^{2}$ Medical Oncology, Azienda Ospedaliero-Universitaria Careggi, Florence, ${ }^{3}$ Department of Medical Biotechnologies, University of Siena, Siena, ${ }^{4}$ Clinical Trials Coordinating Center, Istituto Toscano Tumori, Azienda OspedalieroUniversitaria Careggi, Florence, Italy

*These authors contributed equally to this work

\section{Video abstract}

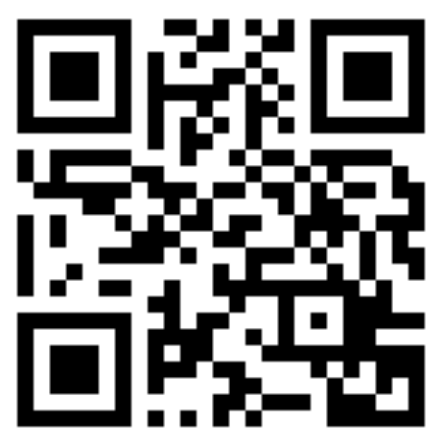

Point your SmartPhone at the code above. If you have a QR code reader the video abstract will appear. Or use: http://youtu.be/w]ZR fRXUwg

Correspondence: Annarosa Arcangeli Department of Experimental and Clinical Medicine, University of Florence, Viale GB Morgagni 50, 50134 Florence, Italy

Tel +39055 275 I283

Fax +39055275 I28।

Email annarosa.arcangeli@unifi.it
Background: The identification of early-stage colorectal cancer (CRC) with high risk of progression is one major clinical challenge, mainly due to lack of validated biomarkers. The aims of the present study were to analyze the prognostic impact of three molecular markers belonging to the ion channels and transporters family: the ether-à-go-go-related gene 1 (hERG1) and the calcium-activated $\mathrm{KCa} 3.1$ potassium channels, as well as the glucose transporter 1 (Glut-1); and to define the impact of adjuvant chemotherapy in conjunction with the abovementioned biomarkers, in a cohort of radically resected stage I-III CRC patients.

Patients and methods: The expressions of hERG1, KCa3.1, and Glut-1 were tested by immunohistochemistry on 162 surgical samples of nonmetastatic, stage I-III CRC patients. The median follow-up was 32 months. The association between biological markers, clinicopathological features, and survival outcomes was investigated by evaluating both disease-free survival and overall survival.

Results: Although no prognostic valence emerged for $\mathrm{KCa} 3.1$, evidence of a negative impact of hERG1 expression on survival outcomes was provided. On the contrary, Glut-1 expression had a positive impact. According to the results of the multivariate analysis, patients were stratified in four risk groups, based on TNM stage and hERG1/Glut-1 expression. After adjusting for adjuvant therapy, stage I and II, Glut-1-negative, and hERG1-positive patients showed the worst survival experience.

Conclusion: This study strongly indicates that the combination of hERG1 positivity and Glut-1 negativity behaves as a prognostic biomarker in radically resected CRC patients. This combination identifies a group of stage I and II CRC patients with a bad prognosis, even worse than that of stage III patients, regardless of adjuvant therapy accomplishment.

Keywords: potassium channels, glucose transporter, biomolecular markers, ion channels, prognostic markers

\section{Introduction}

Colorectal cancer (CRC) is the world's third most common cancer in men and the second most common in women. ${ }^{1}$ Primary treatment for patients without distant metastasis is surgery. Patients with early-stage CRC could expect a long survival with surgery alone, nevertheless $\sim 50 \%$ of stage III and $25 \%$ of stage II will relapse. ${ }^{2}$ Adjuvant chemotherapy is the standard of care for patients with stage III, while the real benefit in stage II is not still clear and the routine use of chemotherapy is not recommended. ${ }^{3}$ As no validated biomarker is available for routinely assessing patients' risk stratification, the decision on whether to accomplish chemotherapy or not for stage II CRC patients currently relies on clinical features as T4, number of lymph nodes analyzed, perforation or obstruction, and grading. ${ }^{4}$ 
Therefore, the identification and validation of novel biomolecular markers, that could support classical clinicopathological parameters in prognostic definition, is one of the upmost challenges in the management of CRC. Such validated biomarkers would in turn help clinicians to identify patients with highest relapse risk and more susceptible to take advantage from adjuvant therapy. Until now, microsatellite instability (MSI) is an important marker to select patients with stage II CRC for adjuvant chemotherapy. Improved survival from adjuvant therapy has been recently demonstrated for patients with proficient DNA mismatch repair (pMMR) tumors, whereas patients with high-level microsatellite (MSI-H) or defective mismatch repair (dMMR) tumors did not show any benefit from fluorouracil (FU)-based therapy. ${ }^{5-8}$ For patients with low-grade MSI ( $85 \%$ of all stage II patients), a promising way to identify groups that could take advantages from adjuvant therapy is ColoPrint (Agendia), an 18-gene expression classifier that identifies early-stage colon cancer patients at higher risk of disease relapse. ${ }^{9,10}$ Other similar multigene assays, for example, Oncotype DX colon cancer assay ${ }^{11}$ and CoIDx, have been evaluated to support clinicians' decisions, providing prognostic and predictive information. Unfortunately, the information obtained from these tests have only a prognostic value, and there is no evidence of predictive value about the potential benefit of adjuvant therapy. A new frontier in this field is represented by some in-silico studies that, making use of large amounts of data originating from available independent data sets, can help in identifying novel potential biomarkers. The predictive role of the transcription factor CDX2 in stage II CRC was identified with a similar approach. Lack of expression of this marker defines a group of patients with high relapse risk, which seems to take advantage from adjuvant therapy, in terms of survival. ${ }^{12}$ More recently, a microRNA-based model was identified, which was capable to enhance in-silico prediction of therapeutic response of individual CRC cases. ${ }^{13}$ Finally, an immunederived $P D-L 1$ gene expression profile helped to identify a subgroup of stage II and III CRC patients with a favorable prognosis that should not receive chemotherapy. ${ }^{14}$

In this study, the prognostic impact of three potential biomarkers belonging to the "ion channels and transporters" family was evaluated: two potassium channels (the ether-à-gogo-related gene 1 [Kv11.1 or hERG1] channel and the "intermediate conductance" calcium-activated KCa3.1 channel, encoded by the KCNN4 gene) and the glucose transporter 1 (Glut-1). hERG1 is a voltage-activated potassium channel belonging to the ether à-go-go (EAG) family, frequently overexpressed in several types of human cancers ${ }^{15-18}$ including
CRC. ${ }^{19-22} \mathrm{KCa} 3.1$ has been shown to be expressed at high levels in many human cancers. ${ }^{23-26}$ Moreover, the impact of $\mathrm{KCa} 3.1$ on cancer cell proliferation, migration, and invasiveness is well described, ${ }^{25-28}$ and the use of Kca3.1 blockers has recently shown promising antitumor effects. ${ }^{29}$ Recently, the expression of $\mathrm{KCa} 3.1$ has also been described in $\mathrm{CRC},{ }^{30}$ but its impact as prognostic or predictive value in CRC patients is still unknown. Glut-1 is a carrier protein being part of the hypoxia pathway, which comprises different biomolecular markers (VEGF-A, CA-IX, and EGFR) switched on when the oxygen levels in tumor tissues decrease. ${ }^{31-33}$ Moreover, its impact in stage I-III CRC patients has been recently investigated and correlated to hERG1 expression. ${ }^{34}$

The aim of the present study was to analyze the prognostic valence of the above three biomarkers in a cohort of nonmetastatic, TNM stage I-III CRC patients, considering the effects of adjuvant therapy on survival. All the markers were tested by immunohistochemistry (IHC) on surgical samples, and the most relevant clinicopathological features were also included in the study.

\section{Patients and methods Patients and sample collection}

For this study, we enrolled a cohort of 162 patients with pathologically confirmed stages I, II, or III colorectal adenocarcinoma and treated with radical surgery at the Department of General Surgery and Surgical Oncology, Azienda Ospedaliero-Universitaria, Careggi, Florence. Patients' cohort was selected without any bias among a group of patients treated surgically at the department from September 2001 to February 2015, excluding patients with chronic hepatitis $\mathrm{C}$ viral infection and those with rectal cancer who had received neoadjuvant radiotherapy or chemotherapy before surgery. After obtaining an informed written consent from each patient, samples of tumor were collected during surgery and treated for IHC analysis, as described later. The study was carried out with approval of the ethics committee of Azienda Ospedaliero-Universitaria Careggi. The classification of adenocarcinomas was conducted under optical microscope, and tumors were staged according to the American Joint Committee on Cancer classification. All patients with disease relapse were treated according to the local guidelines. Moreover, a subgroup of 92 patients (56.8\%) with stage II and III CRC received adjuvant chemotherapy after surgery.

\section{IHC}

For patients enrolled in the study, formalin-fixed, paraffinembedded, $7-\mu \mathrm{m}$ sections were stained by using a commercially 
available kit (PicTure Plus kit and DAB; Zymed, Carlsbad, CA, USA), as described previously. ${ }^{34}$ Briefly, the antigen retrieval for hERG1 and Glut-1 staining was performed by treatment with proteinase $\mathrm{K}(5 \mu \mathrm{g} / \mathrm{mL})$, whereas for $\mathrm{KCa} 3.1$ staining the samples are heated in a microwave oven at $600 \mathrm{~W}$ in citrate buffer $\mathrm{pH} 6.0$ for 15 minutes. Stainings were performed by using antibodies to hERG1 (1:200; monoclonal antibody produced in our laboratory and distributed by Dival Toscana Srl), KCa3.1 (1:2,000, polyclonal rabbit anti-human KCNN4; Sigma-Aldrich, St Louis, MO, USA), and Glut-1 (1:100, polyclonal rabbit anti-human GLUT1; DakoCytomation, Glostrup, Denmark). Tissue slides were analyzed at a total magnification of $40 \times$ field by field, from top left to bottom right, and classified with a scoring system different for each marker. For hERG1, the scoring method described by Lastraioli et $\mathrm{al}^{34}$ was followed: specimens were classified as "score 0 " where no staining was present, specimens with a percentage of neoplastic-stained cells ranging from $1 \%$ to $49 \%$ were classified as "score 1 ," and specimens with a percentage of stained cells $>50 \%$ were classified as "score 2." For the purpose of the analysis, only samples classified with "score 2" (with a high hERG1 expression) were considered "hERG1-positive." For KCa3.1 and Glut-1, only specimens where at least $1 \%$ of marked cells were present, without applying any scoring system, were considered positive..$^{30,34,35}$ Each specimen was analyzed by two independent investigators, and the interobserver agreement was evaluated according to the simple Cohen $\kappa$ of concordance and its 95\% confidence interval (CI). Images were acquired on a Leica DM 4000B microscope with a Leica DFC 320 Camera using Leica QWin software (Leica Microsystems, Milan, Italy).

\section{Statistical methods}

For each patient, the following clinicopathological variables were investigated: age at the intervention, sex, site of tumor, TNM classification, tumor histological grading, mucin content, and adjuvant therapy. Moreover, for each tumor tissue, the expressions of hERG1, KCa3.1, and Glut-1 were also assessed, and each marker was categorized as yes/no with respect to their expression. The association between clinicopathological features and biological markers was evaluated by $\chi^{2}$ and Fisher's exact tests when appropriate. A two-sided $P \leq 0.05$ was considered significant. The impact of each parameter on survival was analyzed by evaluating two variables: disease-free survival (DFS), defined as the time from intervention to death or recurrence of disease, whatever the cause, and overall survival (OS). The Kaplan-Meier inverse method was applied to establish the median follow-up time. ${ }^{36}$ The statistical analysis was performed as described previously. ${ }^{34}$ Briefly, DFS and OS were calculated according to the Kaplan-Meier product-limit method, ${ }^{37}$ first at the univariate analysis, and the Cox proportional hazard model was used to calculate the hazard ratios (HRs) and appropriate 95\% CIs. Subsequently, the independent effect of each parameter on both the survival variables was investigated by a multivariate Cox regression model. As in Lastraioli et al, ${ }^{34}$ starting from a model including all the clinicopathological variables and the biological markers, nonsignificant variables were progressively removed, according to a backward stepwise procedure based on the likelihood ratio test. Finally, for each risk group of patients identified, a Cox proportional hazards model (with the average covariate method) was applied to obtain the nonparametric evaluation of the survivor functions and accompanying HRs, adjusted for adjuvant treatment. Data were analyzed using the statistical software SAS 9.2 (SAS Corporation, Cary, NC, USA).

\section{Results}

\section{Characteristics of the patients' cohort}

Primary tumor samples were collected from 162 patients diagnosed as stage I-III CRC. The clinicopathological features of the patients' cohort are summarized in Table 1. Among 162 patients, 86 (53.1\%) were female and 76 (46.9\%) were male. Patients' age ranged from 40 to 90 years, with a median age of 69 years. Ninety-two patients (56.8\%) received adjuvant chemotherapy after surgery. Tumors were mostly located in the right colon (73), whereas 39 were located in the left, 14 in the transverse colon, and 36 in the rectum.

\section{Analysis of hERGI, KCa3.I, and Glut-I expressions}

In all the samples, the expressions of hERG1 and KCa3.1 potassium channels, as well as that of the glucose transporter Glut-1, by IHC, were investigated using different scoring systems for the three markers (see "Materials and methods"). Figure 1 shows IHC representative pictures relative to samples with different scorings of hERG1 (Figure 1A-C), KCa3.1 (Figure 1D and E), and Glut-1 (Figure 1F and G).

hERG1 turned out to be expressed (ie, score 2) in 40 out of 162 CRC primary tissues $(24.7 \%), \mathrm{KCa} 3.1$ in $56.8 \%$ $(92 / 162)$, and Glut-1 in 34.6\% (56/162) of samples (Table 1). The expressions of hERG1 and Glut-1 were significantly associated $(P=0.001)$, whereas no significant association between $\mathrm{KCa} 3.1$ and the other two biomarkers emerged. 
Table I Univariate analysis of clinicopathological and biomolecular markers for DFS and OS

\begin{tabular}{|c|c|c|c|c|c|c|c|}
\hline \multirow[t]{2}{*}{ Parameter } & \multirow[t]{2}{*}{ Patients, n (\%) } & \multicolumn{3}{|l|}{ DFS } & \multicolumn{3}{|l|}{ os } \\
\hline & & 3-year DFS & HR $(95 \% \mathrm{Cl})$ & $P$-value & 3-year OS & HR $(95 \% \mathrm{Cl})$ & $P$-value \\
\hline Age & & & & 0.98 & & & 0.44 \\
\hline$<70$ years & 85 (52.5\%) & $61.7 \%$ & I (ref) & & $65.2 \%$ & I (ref) & \\
\hline$>70$ years & 77 (47.5\%) & $55.6 \%$ & $0.99(0.60-1.63)$ & & $59.6 \%$ & $1.23(0.72-2.14)$ & \\
\hline Sex & & & & 0.88 & & & 0.59 \\
\hline Female & $86(53.1 \%)$ & $57.2 \%$ & I (ref) & & $62.1 \%$ & I (ref) & \\
\hline Male & 76 (46.9\%) & $59.7 \%$ & I.04 (0.62-I.7I) & & $62.5 \%$ & $1.16(0.67-2.00)$ & \\
\hline Tumor site & & & & 0.94 & & & 0.43 \\
\hline Right colon & 73 (45.1\%) & $57.8 \%$ & I (ref) & & $55.7 \%$ & I (ref) & \\
\hline Transverse colon & 14 (8.6\%) & $51.6 \%$ & $0.83(0.34-2.06)$ & & $51.6 \%$ & $0.96(0.39-2.37)$ & \\
\hline Left colon & $39(24.1 \%)$ & $74.1 \%$ & $0.83(0.44-1.58)$ & & $80.5 \%$ & $0.56(0.26-1.19)$ & \\
\hline Rectum & $36(22.2 \%)$ & $49.5 \%$ & $0.89(0.47-1.68)$ & & $60.5 \%$ & $0.63(0.36-1.45)$ & \\
\hline TNM stage & & & & 0.01 & & & 0.04 \\
\hline Stage I & 32 (19.7\%) & $70.6 \%$ & I (ref) & & $73.0 \%$ & I (ref) & \\
\hline Stage II & 57 (35.2\%) & $67.9 \%$ & $0.98(0.42-2.30)$ & & $77.0 \%$ & $0.88(0.35-2.2 \mathrm{I})$ & \\
\hline Stage III & 73 (45.1\%) & $46.8 \%$ & $2.08(0.97-4.47)$ & & $47.8 \%$ & $1.84(0.81-4.18)$ & \\
\hline Mucin & & & & 0.69 & & & 0.75 \\
\hline No & 120 (74. I\%) & $57.4 \%$ & I (ref) & & $63.3 \%$ & I (ref) & \\
\hline Yes & $42(25.9 \%)$ & $60.6 \%$ & $0.89(0.5 \mathrm{I}-1.56)$ & & $59.7 \%$ & $1.10(0.6 \mathrm{I}-2.00)$ & \\
\hline Histological grading & & & & 0.47 & & & 0.79 \\
\hline GI & 16 (9.9\%) & $57.7 \%$ & I (ref) & & $57.1 \%$ & I (ref) & \\
\hline G2-G3 & $146(90.1 \%)$ & $58.6 \%$ & $0.69(0.25-1.91)$ & & $62.9 \%$ & $0.87(0.3 \mathrm{I}-2.43)$ & \\
\hline Adjuvant & & & & 0.01 & & & 0.01 \\
\hline No & $70(43.2 \%)$ & $76.0 \%$ & I (ref) & & $76.4 \%$ & I (ref) & \\
\hline Yes & $92(56.8 \%)$ & $45.4 \%$ & $2.78(1.58-4.88)$ & & $51.6 \%$ & $2.34(1.28-4.28)$ & \\
\hline hERGI & & & & 0.26 & & & 0.17 \\
\hline Negative & $122(75.3 \%)$ & $60.4 \%$ & I (ref) & & $64.5 \%$ & I (ref) & \\
\hline Positive & $40(24.7 \%)$ & $51.2 \%$ & $1.38(0.78-2.46)$ & & $54.0 \%$ & $1.54(0.83-2.85)$ & \\
\hline KCa3.I & & & & 0.55 & & & 0.86 \\
\hline Negative & $70(43.2 \%)$ & $57.8 \%$ & I (ref) & & $58.0 \%$ & I (ref) & \\
\hline Positive & 92 (56.8\%) & $59.3 \%$ & $0.86(0.5 I-1.42)$ & & $64.9 \%$ & $1.05(0.60-1.84)$ & \\
\hline Glut-I & & & & 0.02 & & & 0.01 \\
\hline Negative & $106(65.4 \%)$ & $49.5 \%$ & I (ref) & & $54.9 \%$ & I (ref) & \\
\hline Positive & $56(34.6 \%)$ & $78.0 \%$ & $0.5 \mathrm{I}(0.28-0.91)$ & & $78.2 \%$ & $0.410(0.21-0.80)$ & \\
\hline
\end{tabular}

Note: Statistically significant parameters are highlighted in bold.

Abbreviations: $\mathrm{Cl}$, confidence interval; DFS, disease-free survival; HR, hazard ratio; OS, overall survival.

\section{Association analysis}

The associations between the expressions of the three biomolecular markers and the clinicopathological characteristics of the patients were analyzed. No significant association emerged between the expressions of the two potassium channels and clinicopathological characteristics such as age, TNM stages, sex, histological grading, and adjuvant therapy. KCa3.1 was mainly expressed in mucinous CRC primary samples $(71.4 \%$ mucinous tumors vs $52.5 \%$ nonmucinous tumors; $P=0.045$ ), and an association, although not significant, was found between hERG1 and tumor site, with the channel more expressed in transverse and left colon $(P=0.267)$.

Glut-1 expressed more in patients aged $<70$ years $(42.4 \%$ vs $26 \% ; P=0.029)$ and in left and transverse colon $(27.4 \%$, $42.9 \%, 53.9 \%$, and $25 \%$ for right colon, transverse colon, left colon, and rectum, respectively; $P=0.019)$. Glut-1 was less frequently detected in mucinous tumors $(P=0.088)$.

\section{Impact on survival outcomes}

The impact on survival was analyzed evaluating both DFS and OS. Patients were followed up for a median time of 32 months. Thirty of 162 patients (18.5\%) had a disease relapse and $32(19.8 \%)$ died during follow-up. The univariate analysis (Table 1) showed that TNM stage, adjuvant therapy, and Glut-1 expression have a significant impact on DFS and OS: TNM stage III and adjuvant therapy emerged as indicators of worse prognosis, whereas Glut-1 had a positive impact on survival.

The multivariate analysis (Table 2) confirmed the trends of stage III TNM, adjuvant therapy, and Glut-1 expression and also showed a significant negative impact of hERG1 expression on both DFS and OS. For KCa3.1, both univariate 

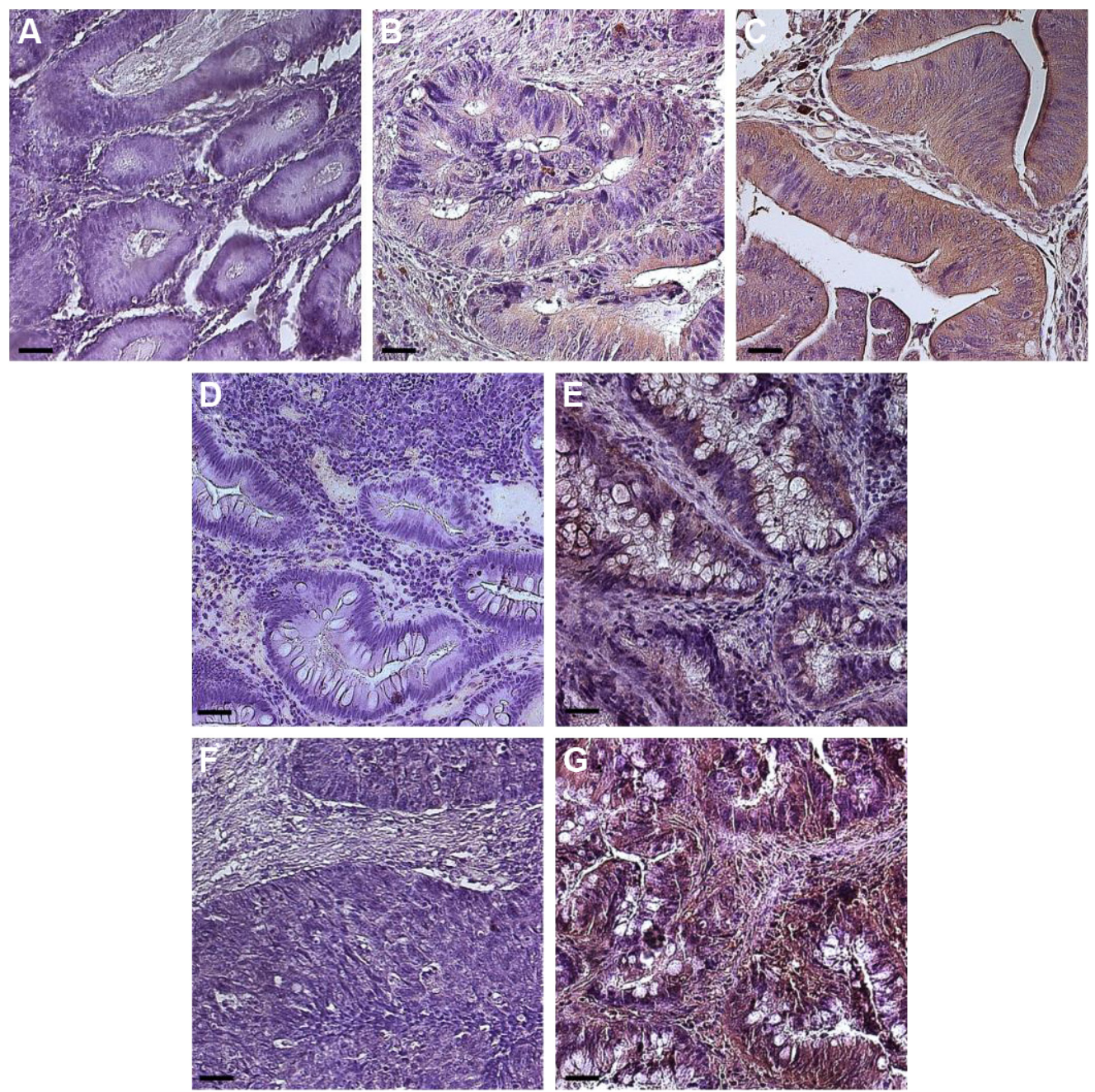

Figure I Immunohistochemical scoring for all markers in CRC primary samples.

Notes: (A-C) Representative examples of hERGI scoring in CRC specimens using the anti-hERGI monoclonal antibody: (A) score 0 (0\% of positive cells), (B) score I ( $1 \%-49 \%$ of positive cells per microscopic field), and (C) score 2 (>50\% of positive cells per microscopic field). Note that only samples belonging to score 2 were considered positive. ( $\mathbf{D}$ and $\mathbf{E})$ Representative example of $\mathrm{KCa}$.I scoring: negative (D) and positive (E) representative $\mathrm{CRC}$ specimens. (F and $\mathbf{G}) \mathrm{Glut}$ - I scoring in representative $\mathrm{CRC}$ specimens: (F) negative and (G) positive samples. Original magnification: $200 \times$. Scale bars: $50 \mu \mathrm{m}$.

Abbreviations: CRC, colorectal cancer; IHC, immunohistochemistry.

and multivariate analyses did not show any statistically relevant association with the survival outcomes. For risk stratification analysis, all the variables that did not show any significant independent effect on DFS and OS were progressively removed. After adjusting for adjuvant treatment, this analysis led to stratification of the patients into four different risk groups, based on TNM stages and hERG1/Glut-1 expression. Three groups encompass TNM stage I and II patients, who are further subdivided on the basis of the expression of hERG1 and Glut-1, and the fourth group comprises stage III patients independently on the molecular phenotype. As shown in Figure 2 and Table 3, the group comprising Glut1-negative and hERG1-positive stage I and II patients had the worst survival experience.

\section{Discussion}

The present study was aimed at identifying novel biomolecular markers to be employed in the clinical practice for the identification of high-risk early-stage CRC patients, for further selection of treatment options. The prognostic impact of two potassium channels hERG1 and $\mathrm{KCa} 3.1$, as well as that of Glut-1, in a cohort of 162 surgically resected, stages I-III CRC patients was analyzed. It is evident that, independent of adjuvant treatment effect, 1) three main variables significantly impact on survival (both OS and DFS): TNM (negative when stage III), hERG1 (negative), and Glut-1 (positive); 2) hERG1 positivity and Glut-1 negativity serve to identify a subset of stage I and II CRC patients whose survival curves are worse than those of stage III CRC patients. 
Table 2 Multivariate analysis of factors related to DFS and OS (by the Cox's regression model)

\begin{tabular}{|c|c|c|c|c|}
\hline \multirow[t]{2}{*}{ Parameter } & \multicolumn{2}{|l|}{ DFS } & \multicolumn{2}{|l|}{ OS } \\
\hline & HR (95\% CI) & $P$-value & HR (95\% Cl) & $P$-value \\
\hline TNM stage & & 0.0110 & & 0.0060 \\
\hline I & I (ref) & & I (ref) & \\
\hline II & $0.43(0.17-1.10)$ & & $0.39(0.14-1.08)$ & \\
\hline III & I. $16(0.48-2.79)$ & & $1.16(0.46-2.92)$ & \\
\hline hERGI & & 0.0127 & & 0.0058 \\
\hline Negative & I (ref) & & I (ref) & \\
\hline Positive & $2.57(1.40-4.73)$ & & $2.75(1.43-5.29)$ & \\
\hline $\mathrm{KCa} 3 . \mathrm{I}$ & & 0.0708 & & 0.3109 \\
\hline Negative & I (ref) & & I (ref) & \\
\hline Positive & $0.59(0.34-1.04)$ & & $0.72(0.38-1.35)$ & \\
\hline Glut-I & & 0.0004 & & $<0.0001$ \\
\hline Negative & I (ref) & & I (ref) & \\
\hline Positive & $0.32(0.17-0.60)$ & & $0.24(0.12-0.49)$ & \\
\hline Adjuvant & & 0.0001 & & 0.0012 \\
\hline No & I (ref) & & I (ref) & \\
\hline Yes & $3.42(1.75-6.67)$ & & $3.03(1.50-6.13)$ & \\
\hline
\end{tabular}

Abbreviations: $\mathrm{Cl}$, confidence interval; DFS, disease-free survival; $\mathrm{HR}$, hazard ratio; OS, overall survival.

Nowadays, the identification of novel prognostic biomarkers is mandatory, in order to help clinicians in risk stratification and decision making in the treatment of CRC patients with early-stage, in particular stage II, disease. ${ }^{38}$ Indeed, it is well known that a subgroup of stage II patients, usually not treated with adjuvant chemotherapy, recurs after surgery. ${ }^{39}$ Those stage II patients whose disease recurs would benefit from adjuvant chemotherapy, like those with a TNM stage III disease. Hence, clinicians continue to grapple with the problem of determining those stage II patients most likely to derive benefits from adjuvant chemotherapy, in order to improve the outcomes in this patient population and, in the meantime, avoid potentially toxic overtreatments. The most important parameters among a handful of prognostic variables that are considered for treatment choices are only tumor stage, tumor grade, and MSI. ${ }^{40,41}$

In the present study, the expression and the prognostic impact of two potassium channels, hERG1 and KCa3.1, whose aberrant expression has been strongly associated to many human cancer, including CRC, were investigated. ${ }^{15-30}$ The expression of Glut-1, another cancer biomarker, belonging to the hypoxia signaling pathway was also investigated. ${ }^{31-33}$ Moreover, a previous observation, obtained in a pilot study, demonstrated a significant association and prognostic valence for the combined hERG1 and Glut-1 expressions in CRC patients. ${ }^{34}$

The expression of KCa3.1 channel was detected in $>50 \%$ of CRC primary samples. Unexpectedly, our data did not show any diagnostic or prognostic relevance for $\mathrm{KCa} 3.1$ expression, at least in nonmetastatic CRC, even though its role in driving tumor progression and its association to poor prognosis in other types of human cancer are well recognized..$^{23-30}$

On the contrary, hERG1 expression displayed a negative impact on survival outcomes that, although nonsignificant at the univariate analysis, reached significance in the multivariate model. Most parameters losing their valence, when
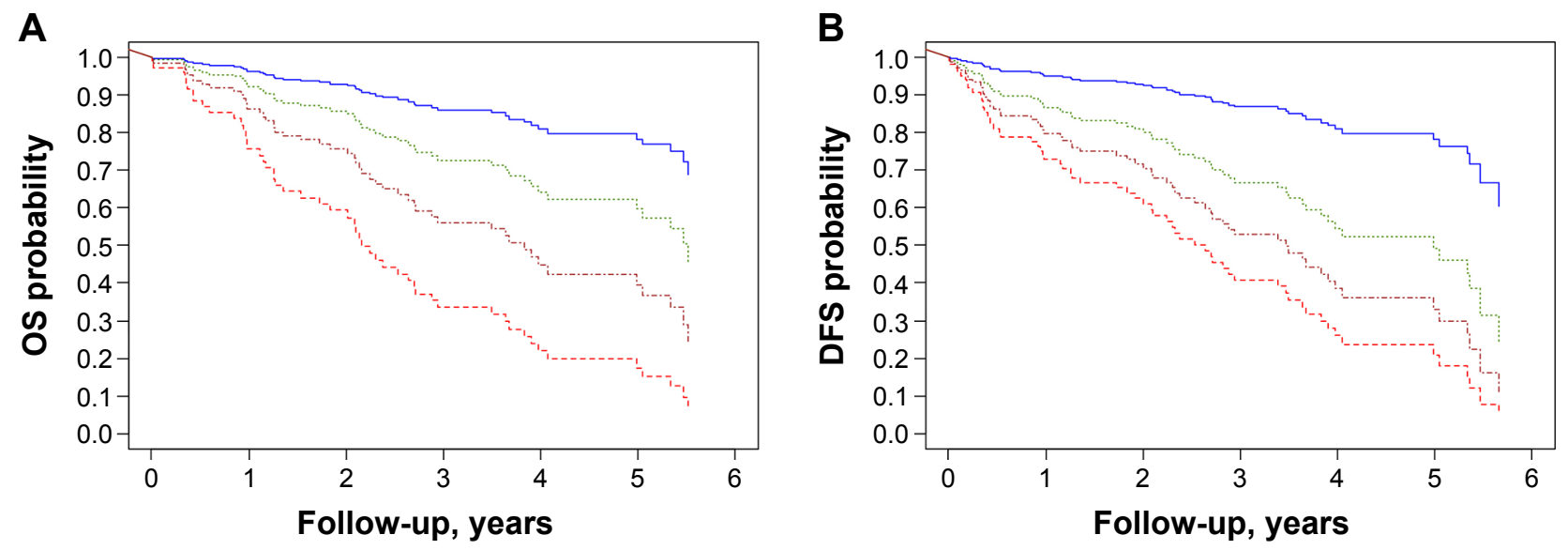

\begin{tabular}{|c|c|}
\hline - TNM I/II Glut-1+(N=27) & -.. TNM I/II Glut-1-hERG1+ $(\mathrm{N}=11)$ \\
\hline$\ldots$ TNM I/II Glut-1-hERG1- $(\mathrm{N}=51)$ & $\ldots$ TNM III $(\mathrm{N}=73)$ \\
\hline
\end{tabular}

Figure 2 Kaplan-Meier curves of overall and disease-free survival according to different combinations of tumor characteristics (TNM stage, Glut-I, and hERGI status). Notes: Kaplan-Meier plots of (A) overall survival (OS) and (B) disease-free survival (DFS) probabilities for four different groups are reported. Blue curve indicates stage I and II Glut-I-positive samples (27 patients, I6.7\%); red curve, stage I and II Glut-I-negative and hERG I-positive samples (eleven patients, 6.8\%); green curve, stage I and II Glut-I-negative and hERG I-negative samples (5I patients, 31.5\%); brown curve, stage III samples (73 patients, $45.1 \%$ ). 
Table 3 Association between risk groups and survival outcomes adjusted for adjuvant treatment (by means of Cox's proportional hazard model)

\begin{tabular}{|c|c|c|c|c|c|c|c|}
\hline \multirow[t]{2}{*}{ Parameter } & \multirow[t]{2}{*}{ Patients (n) } & \multicolumn{3}{|l|}{ DFS } & \multicolumn{3}{|l|}{ OS } \\
\hline & & 3-year DFS & HR (95\% CI) & $P$-value & 3-year OS & HR (95\% Cl) & $P$-value \\
\hline TNM III & 73 & $52.9 \%$ & I (ref) & 0.032 & $56.2 \%$ & I (ref) & 0.021 \\
\hline TNM I/II Glut-I+ & 27 & $86.8 \%$ & $0.22(0.05-0.95)$ & & $85.9 \%$ & $0.26(0.06-1.14)$ & \\
\hline TNM I/II Glut-I- hERG I+ & 11 & $40.8 \%$ & I.4I (0.58-3.40) & & $33.7 \%$ & $1.89(0.77-4.70)$ & \\
\hline TNM I/II Glut-I- hERG I- & 51 & $66.6 \%$ & $0.64(0.35-1.17)$ & & $72.6 \%$ & $0.56(0.28-I . I I)$ & \\
\hline
\end{tabular}

Abbreviations: $\mathrm{Cl}$, confidence interval; DFS, disease-free survival; $\mathrm{HR}$, hazard ratio; OS, overall survival.

analyzed under the multivariate models, is an unusual feature. However, the negative impact of hERG1 on prognosis is not surprising, as the impact of hERG1 on tumor progression has been proven by several published papers. ${ }^{15-22}$

Glut-1 showed the strongest correlation with clinicopathological features. In fact, statistically relevant correlations emerged with age (more expressed in $<70$ years cluster) and tumor site (more expressed in left colon and transverse). Furthermore, Glut-1 positively impacted on the survival at both the univariate and multivariate analyses. Such a positive impact is apparently in contrast with the common view considering Glut-1, being a hypoxia marker, as a tumor progression factor. ${ }^{31-33,42,43}$ As discussed in Lastraioli et al, ${ }^{34}$ it is believed that the highest Glut-1 expression occurs at a preangiogenic phase of tumor progression, and its disappearance marks the onset of angiogenesis, the true progression step underlying the acquisition of full malignancy in CRC.

Overall, four variables expressed a significant impact on survival: TNM stages, therapy, hERG1, and Glut-1 expressions. The negative impact on the survival of TNM is well defined. ${ }^{44}$ The negative prognostic role of adjuvant therapy may appear contradictory, as it was developed with the purpose of improving survival. Until now, this negative impact has been misleading, because it depends more on the clinical characteristics of the group of patients (either stage III or II) who were selected for treatment than on the effects of treatments.

The most novel and relevant result emerging from the present study was that a strong hERG1 expression, combined with the lack of Glut-1 expression, is associated with significant worsening of the prognosis of surgically resectable early stages of CRC patients. The present risk analysis, besides confirming a previous pilot study, ${ }^{34}$ led to an even stronger evidence that the outcome of hERG1-positive/Glut-1-negative patients is worse than that of stage III patients.

On the whole, based on the results reported here, it has been proposed that an IHC-based test addressing hERG1 and Glut-1 detection (hERG1/Glut-1 test) could be used in stage II CRC patients to determine the individual risk of cancer recurrence. Alone, or in conjunction with MSI or CDX2 analysis, the hERG1/Glut-1 test could accompany, or even substitute, more complex biomolecular tests. Furthermore, it has been proposed to accomplish an appropriately designed study to confirm the predictive potential of hERG1 positivity and Glut-1 negativity. Once validated in a clinical study, the hERG1/Glut-1 test would contribute to identify those stage II patients with likelihood of benefit from adjuvant chemotherapy.

\section{Acknowledgments}

The authors thank Lapo Bencini, Marco Farsi, Ilenia Bartolini, and Andrea Coratti, all of General Surgery and Surgical Oncology, Azienda Ospedaliero-Universitaria Careggi, to collect and provide samples. This work was supported by Associazione Italiana per la Ricerca sul Cancro (AIRC; Grant number 15627 to $\mathrm{AA})$.

\section{Disclosure}

The authors report no conflicts of interest in this work.

\section{References}

1. Siegel R, Desantis C, Jemal A. Colorectal cancer statistics, 2014. $C A$ Cancer J Clin. 2014;64(2):104-117.

2. Haggar FA, Boushey RP. Colorectal cancer epidemiology: incidence, mortality, survival, and risk factors. Clin Colon Rectal Surg. 2009;22(4):191-197.

3. Dienstmann R, Salazar R, Tabernero J. Personalizing colon cancer adjuvant therapy: selecting optimal treatments for individual patients. $J$ Clin Oncol. 2015;33(16):1787-1796.

4. Dunn KB, Trudel JL. Colorectal cancer: adjuvant therapy. In: Beck DE, Roberts PL, Saclarides TJ, Senagore AJ, Stamos MJ, Nasseri Y, editors. The ASCRS Textbook of Colon and Rectal Surgery. New York: Springer; 2011:773-782.

5. Thibodeau SN, Bren G, Schaid D. Microsatellite instability in cancer of the proximal colon. Science. 1993;260:816-819.

6. Kim GP, Colangelo LH, Wieand HS, et al. for National Cancer Institute. Prognostic and predictive roles of high degree microsatellite instability in colon cancer: a National Cancer Institute - National Surgical Adjuvant Breast and Bowel Project Collaborative Study. J Clin Oncol. 2007;25(7):767-772.

7. Sargent DJ, Marsoni S, Monges G, et al. Defective mismatch repair as a predictive marker for lack of efficacy of fluorouracil-based adjuvant therapy in colon cancer. J Clin Oncol. 2010;28(20):3219-3226. 
8. Sinicrope FA, Foster NR, Thibodeau SN, et al. DNA mismatch repair status and colon cancer recurrence and survival in clinical trials of 5-fluorouracil-based adjuvant therapy. J Natl Cancer Inst. 2011;103(11):863-875.

9. Salazar R, Roepman P, Capella G, et al. Gene expression signature to improve prognosis prediction of stage II and III colorectal cancer. J Clin Oncol. 2011;29(1):17-24.

10. Kopetz S, Tabernero J, Rosenberg R, et al. Genomic classifier ColoPrint predicts recurrence in stage II colorectal cancer patients more accurately than clinical factors. Oncologist. 2015;20(2):127-133.

11. You YN, Rustin RB, Sullivan JD. Oncotype DX $\left({ }^{\circledR}\right)$ colon cancer assay for prediction of recurrence risk in patients with stage II and III colon cancer: a review of the evidence. Surg Oncol. 2015;24(2):61-66.

12. Dalerba $P$, Sahoo D, Paik S, et al. CDX2 as a prognostic biomarker in stage II and stage III colon cancer. N Engl J Med. 2016;374(3):211-222.

13. Li J, Mansmann UR. A microRNA molecular modeling extension for prediction of colorectal cancer treatment. BMC Cancer. 2015;15:472.

14. Dunne PD, McArt DG, O'Reilly PG, et al. Immune-derived PD-L1 gene expression defines a subgroup of stage II/III colorectal cancer patients with favorable prognosis that may be harmed by adjuvant chemotherapy. Cancer Immunol Res. 2015;3(10):1158-1164.

15. Jehle J, Schweizer PA, Katus HA, Thomas D. Novel roles for hERG $\mathrm{K}(+)$ channels in cell proliferation and apoptosis. Cell Death Dis. 2011;2:e193.

16. Lastraioli E, Lottini T, Bencini L, Bernini M, Arcangeli A. hERG1 potassium channels: novel biomarkers in human solid cancers. Biomed Res Int. 2015;2015:896432.

17. Arcangeli A, Crociani O, Lastraioli E, Masi A, Pillozzi S, Becchetti A. Targeting ion channels in cancer: a novel frontier in antineoplastic therapy. Curr Med Chem. 2009;16:66-93.

18. Arcangeli A. Expression and role of hERG channels in cancer cells. Novartis Found Symp. 2005;266:225-232; discussion 232-234.

19. Lastraioli E, Guasti L, Crociani O, et al. herg1 gene and HERG1 protein are overexpressed in colorectal cancers and regulate cell invasion of tumor cells. Cancer Res. 2004;64(2):606-611.

20. Crociani O, Zanieri F, Pillozzi S, et al. hERG1 channels modulate integrin signaling to trigger angiogenesis and tumor progression in colorectal cancer. Sci Rep. 2013;3:3308.

21. Ousingsawat J, Spitzner M, Puntheeranurak S, et al. Expression of voltage-gated potassium channels in human and mouse colonic carcinoma. Clin Cancer Res. 2007;13(3):824-831.

22. Dolderer JH, Schuldes CH, Bockhorn A, et al. HERG1 gene expression as a specific tumor marker in colorectal tissues. Eur J Surg Oncol. 2010;36(1):72-77.

23. D’Amico M, Gasparoli L, Arcangeli A. Potassium channels: novel emerging biomarkers and targets for therapy in cancer. Recent Pat Anticancer Drug Discov. 2013;8:53-65.

24. Weaver AK, Bomben VC, Sontheimer H. Expression and function of calcium-activated potassium channels in human glioma cells. Glia. 2006;54:223-233.

25. Haren N, Khorsi H, Faouzi M, Ahidouch A, Sevestre H, OuadidAhidouch $\mathrm{H}$. Intermediate conductance $\mathrm{Ca} 2+$ activated $\mathrm{K}+$ channels are expressed and functional in breast adenocarcinomas: correlation with tumour grade and metastasis status. Histol Histopathol. 2010; 25(10):1247-1255.

OncoTargets and Therapy

\section{Publish your work in this journal}

OncoTargets and Therapy is an international, peer-reviewed, open access journal focusing on the pathological basis of all cancers, potential targets for therapy and treatment protocols employed to improve the management of cancer patients. The journal also focuses on the impact of management programs and new therapeutic agents and protocols on

Submit your manuscript here: http://www.dovepress.com/oncotargets-and-therapy-journal
26. Wang ZH, Feng YJ, Su M, Yi XF. Intermediate-conductance-Ca2+activated $\mathrm{K}+$ channels are overexpressed in endometrial cancer and involved in regulating proliferation of endometrial cancer cells. Zhonghua Fu Chan Ke Za Zhi. 2007;42:111-115.

27. Schmidt J, Friebel K, Schönherr R, Coppolino MG, Bosserhoff AK. Migration-associated secretion of melanoma inhibitory activity at the cell rear is supported by KCa3.1 potassium channels. Cell Res. 2010;20:1224-1238.

28. Rabjerg M, Oliván-Viguera A, Hansen LK, et al. High expression of $\mathrm{KCa} 3.1$ in patients with clear cell renal carcinoma predicts high metastatic risk and poor survival. PLoS One. 2015;10(4):e0122992.

29. Liu Y, Zhao L, Ma W, et al. The blockage of KCa3.1 channel inhibited proliferation, migration and promoted apoptosis of human hepatocellular carcinoma cells. J Cancer. 2015;6(7):643-651.

30. Lai W, Liu L, Zeng Y, et al. KCNN4 channels participate in the EMT induced by PRL-3 in colorectal cancer. Med Oncol. 2013;30(2):566.

31. McGuire BB, Fitzpatrick JM. Biomarkers in renal cell carcinoma. Curr Opin Urol. 2009;19:441-446.

32. Szablewski L. Expression of glucose transporters in cancers. Biochim Biophys Acta. 2013;1835:164-169.

33. Stewart PA, Parapatics K, Welsh EA, et al. A pilot proteogenomic study with data integration identifies MCT1 and GLUT1 as prognostic markers in lung adenocarcinoma. PLoS One. 2015;10(11):e0142162.

34. Lastraioli E, Bencini L, Bianchini E, et al. hERG1 channels and Glut-1 as independent prognostic indicators of worse outcome in stage I and II colorectal cancer: a pilot study. Transl Oncol. 2012;5(2):105-112.

35. Cooper R, Sarioğlu S, Sökmen S, et al. Glucose transporter-1 (GLUT-1): a potential marker of prognosis in rectal carcinoma? Br J Cancer. 2003;89(5):870-876.

36. Schemper M, Smith TL. A note on quantifying follow-up in studies of failure time. Control Clin Trials. 1996;17:343-346.

37. Kaplan EL, Meier P. Non parametric estimation from incomplete observations. J Am Stat Assoc. 1958;53:457-481.

38. Compton C, Fenoglio-Preiser CM, Pettigrew N, Fielding LP. American Joint Committee on Cancer Prognostic Factors Consensus Conference: Colorectal Working Group. Cancer. 2000;88:1739-1757.

39. Liefers GJ, Cleton-Jansen AM, van de Velde CJ, et al. Micrometastases and survival in stage II colorectal cancer. $N$ Engl $J$ Med. 1998;339(4):223-228.

40. Bardia A, Loprinzi C, Grothey A, et al. Adjuvant chemotherapy for resected stage II and III colon cancer: comparison of two widely used prognostic calculators. Semin Oncol. 2010;37:39-46.

41. Tournigand C, de Gramont A. Chemotherapy: is adjuvant chemotherapy an option for stage II colon cancer? Nat Rev Clin Oncol. 2011;8:574-576.

42. Haber RS, Rathan A, Weiser KR, et al. GLUT1 glucose transporter expression in colorectal carcinoma: a marker for poor prognosis. Cancer. 1998;83(1):34-40.

43. Airley R, Evans A, Mobasheri A, Hewitt SM. Glucose transporter Glut-1 is detectable in peri-necrotic regions in many human tumor types but not normal tissues: study using tissue microarrays. Ann Anat. 2010;192:133-138.

44. Compton CC. Colorectal carcinoma: diagnostic, prognostic, and molecular features. Mod Pathol. 2003;16(4):376-388.

\section{Dovepress}

patient perspectives such as quality of life, adherence and satisfaction. The manuscript management system is completely online and includes a very quick and fair peer-review system, which is all easy to use. Visit http://www.dovepress.com/testimonials.php to read real quotes from published authors. 